\title{
Practical Application of International Standards and Guidelines to Address Ethical Challenges in Clinical Trials
}

\author{
Juntra Karbwang ${ }^{1}$ and Kesara Na-Bangchang ${ }^{2}$
}

${ }^{1}$ Department of Clinical Product Development, Institute of Tropical Medicine, Nagasaki University, Nagasaki, Japan

2International College of Medicine, Thammasat University, Pathumthani, Thailand

\begin{abstract}
The aim of this article is to articulate how the international standards in clinical trials can assist in addressing ethical challenges when designing, conducting, analyzing, and reporting clinical trial. The ethical issues related to technical aspects of protocol are commonly seen in study design, selection of subjects, selection of control group, and estimation of sample size. The appropriate study design should be chosen to provide the desired information. Validity of research results depends on the extent to which investigators have been able to avoid all possible sources of bias. The techniques used to minimize biases are randomization, blinding, and the use of control group. Ethics committee members are required to evaluate whether the chosen study design is appropriate for the type of study under the circumstances and whether biases have been sufficiently managed.
\end{abstract}

Keywords: Research ethics; Clinical trial

\section{Introduction}

When generating clinical data to support drug/vaccine marketing registration, it is imperative to design, conduct, analyze, and report them in accordance with international ethical and scientific standards that are accepted by drug/vaccine regulators in the countries. Series of reports from the International Conference on Harmonization of Technical Requirements for Registration of Pharmaceuticals for Human Use (ICH) have been widely accepted by most of regulators throughout the world. The principles and requirements layout in these reports should be followed when generating data to support application for marketing registration. Most of the time, the only report that is well known to investigators is the Good Clinical Practice guidelines (ICH GCP) [1]. This report focuses on the responsibilities of ethics committee, investigator and sponsor when conducting a clinical trial. It gives a framework and standards for conducting clinical trial, compliance with these standards will provide public assurance that the trial subjects are protected and the data are credible [1]. In order to be in compliance with ICH GCP, consultation with other associated documents such as ICH 8 (General Considerations for Clinical Trials) [2], ICH 9 (Statistical Principles for Clinical Trials) [3], ICH 10 (Choice of Control Group and Related Issues in Clinical Trials) [4], may be necessary. When the study involves children, ICH 11 (Clinical Investigation of Medicinal Products in Pediatric Population) [5] should be consulted. In addition, the Declaration of Helsinki [6] and the Council of Medical Science (CIOMS) guidelines [7] are very useful resources for ethical decision making when designing clinical trials.

The aim of this paper is to discuss how to use the information provided in various ICH guidelines in an attempt to addressing common ethical challenges when designing clinical trials.

\section{Principles of ICH GCP}

The clinical trial must be conducted in accordance with basic ethical principles that have their origins in the Declaration of Helsinki. Prior to initiating any clinical trial, foreseeable risks from research procedures should be identified and weighed against any anticipated benefit(s) $[1,6,7]$. The safety information of investigational product and clinical trial procedures should be supported by sufficient information from pre-clinical studies and clinical studies (if available) [1,6]. Clinical trials should be designed, conducted, analyzed, and reported according to sound scientific and ethical principles to ensure the validity of trial data and to achieve trial objectives $[1,6]$. The design and performance of each trial should be clearly described in a clinical trial protocol $[1,6]$. The Declaration of Helsinki requires that a statement of the ethical concerns be included in the protocol and it should indicate how these concerns have been addressed [6]. The protocol must receive an approval or favorable opinion from independent ethics committee prior to initiation of the trial $[1,6]$.

The clinical trial should be conducted by qualified investigators and in accordance with approved protocol $[1,6]$. All trial subjects should receive sufficient relevant information to support their decision making in participating in the trial. The informed consent must be valid (i.e., full disclosure of information, competency and voluntariness of subjects) [8]. Throughout the clinical trial, emerging information on safety should be reviewed and evaluated by qualified experts and ethics committee to assess their implications for the safety of the trial subjects [1]. As a consequence of such findings, the protocol and/or informed consent may require appropriate modification in a timely fashion [1,9] to maintain the safety of trial subjects. There is a need for continued review of risk/benefit ratio at regular interval $[1,6,9]$. The trial should be continued only if the benefit-risk ratio remains favorable. The privacy and confidentiality of the trial subjects should be respected throughout the study and post trial period $[1,9,10]$. All trial information should be recorded, handled and stored in a manner that allows its accurate reporting, interpretation, and verification [1] The investigator and sponsor share the responsibility of protecting subjects in clinical trials, together with the Institutional Review Board/ Independent Ethics Committee [1].

*Corresponding author: Kesara Na-Bangchang, International College of Medicine, Thammasat University, Pathumthani, Thailand, Tel: +818027846053; Fax: +818027846055; E-mail: karbwangj@nagasaki-u.ac.jp

Received February 04, 2013; Accepted April 20, 2013; Published May 03, 2013

Citation: Karbwang J, Na-Bangchang K (2013) Practical Application of International Standards and Guidelines to Address Ethical Challenges in Clinical Trials. J Clinic Res Bioeth 4: 144. doi:10.4172/2155-9627.1000144

Copyright: (c) 2013 Karbwang J, et al. This is an open-access article distributed under the terms of the Creative Commons Attribution License, which permits unrestricted use, distribution, and reproduction in any medium, provided the original author and source are credited. 


\section{Ethical Considerations in Clinical Trial}

Ethical issues in clinical trials should be identified and addressed when developing a clinical study protocol. Ethical standards have been defined in relation to scientific design of the study (i.e., minimizing risks and maximizing benefits to create a favourable risk-benefit ratio), appropriate selection and recruitment of study participants, adequacy of medical care during the study and post-trial periods, compensation for any inconvenience and injury associated with the trial, protection of participant privacy and confidentiality, provision for proper informed consent, and a prior review and approval by Ethics Committees (ECs). Ethical issues related to technical aspects of the protocol are commonly seen in the study design of a specific trial in a specific circumstance, selection of control group, selection of subjects and estimation of sample size. The discussion will confine to these four aspects.

\section{Study design}

The purpose of clinical trial is to evaluate the safety and efficacy of the intervention product (drug or vaccine). The data collected can be independent data where each group of subjects receives different interventions, or paired data where the evaluation on each individual subject is carried out more than once. The appropriate study design should be chosen to provide the desired information. The validity of the results depends on the extent to which investigators have been able to avoid all possible sources of bias $[11,12]$. The techniques commonly used to minimize bias are randomization, blinding, and the use of control group $[2,11,12]$.

Randomization is the mean to ensure the independent allocation of subjects to the trial; all subjects have the same chance to treatment assignment. Randomization removes any chance of allocation bias. For randomization to be ethical, the stage of 'equipoise' is required.

Blinding is an important means of reducing or minimizing the risk of biased study outcomes. The blinding can be a single blind (subjects do not know the treatment assignment) or double blind (both subject and investigator do not know the treatment assignment).

Using control group will allow an objective evaluation of the effect of intervention, if any. However, selection of control group should be appropriate with adequate numbers of subjects included to achieve the study objectives.

Examples of study design include parallel group, cross-over, factorial, dose escalation, and fixed dose-dose response.

\section{Selection of control group}

Trials should have an adequate control group. Comparisons may be made with placebo, no treatment, and different active controls or with different doses of the drug/vaccine under investigation. The choice of the comparator depends, among other things, on the objective of the trial, the disease, and the context of the trial $[2,13]$. Choosing control group for clinical trial remains a challenge, particularly when carrying out the trial in developing countries where the best treatment is not available [13] or standard treatment has serious side effects [14]. However, when the best current treatment is not available locally, the best nationally available treatment or the so called 'established effective treatment' may be accepted, provided that it has acceptable side effects [7]. Furthermore, research designs that compare new treatment with the best current proven treatment may be of little relevance in countries where such treatments are not available due to their high costs [15].

The use of placebo or no treatment is limited to situations where no current proven treatment exists or where it is necessary for scientifically sound methodological reasons $[4,6,16]$. The sound methodological reasons should include the following conditions: the disease with a high placebo response rate, the disease with a waxing and waning characteristic; and current proven treatment is known to have severe toxicity that patients refuse to use it $[4,14,16]$.The ethical justification should be provided in the protocol [17] and the study should be carefully monitored and rescue treatment should be readily available when required [14]. As a general rule, it is not ethically acceptable to use placebo-controlled trial design when effective therapy that is known to prevent death or irreversible morbidity exists $[4,6,7,13,14,16]$.

There are other situations where placebo could be ethically justifiable such as those existing treatment is not accepted uniformly as standard (i.e. insufficient evidence of efficacy to out-weight the potential risks), those with known evidence of long-term treatment but uncertain on short-term treatment, and those with efficacy evidence that limited to symptoms but concern exists for potentially important long-term effects [13].

When therapy is targeted at less serious conditions, placebo control group may be used as internal evidence of assay sensitivity. ${ }^{\star}$ In a threearm trial design, placebo and active control are used to assess if the test drug is ineffective or the trial lacks assay sensitivity [4]. When a difference is demonstrated, it is interpretable without reference to external findings. For example, a study with an objective of assessing efficacy of a drug as anti-relapse in Plasmodium vivax malaria was proposed to compare with an active control. When test drug shows the same relapse rate with the active control, the interpretation of the result could be that the test drug has activity against the relapse. However, when a placebo group is added as another control group and it shows no difference in relapse rate, the interpretation would be that the test drug is not effective. On the other hand, if the placebo group has significant higher relapse rate, then the test drug is effective. This is due to the fact that not all patients with $P$. vivax will have relapse episode even without anti-relapse treatment. In this condition, placebo is used as internal evidence of assay sensitivity. In a dose-response study, placebo as an additional group permits an estimate of the total pharmacologically mediated effect of test doses [4]. When all doses produce similar effects, placebo group can assist in the interpretation that they are equally effective or equally ineffective. In any placebocontrol study, unbalanced randomization (e.g., 2:1 or 3:1 study drug to placebo) is recommended [4].

\section{Selection of subjects}

In early trials, the subjects may be limited to a narrow range with strict selection criteria $[2,11]$. When drug/vaccine development proceeds, the subject should be broadened to reflect the target population. For a drug expected to be used in children, it is ethically appropriate to begin with older children before extending the trial to younger children and then infants [2,7]. For vulnerable subjects to be included in the clinical trial, ethical justification of their involvement is required to affirm that the research could not be carried out equally well in less vulnerable subjects [7]. As a general principle, the subjects should not be concurrently enrolled in more than one clinical trial or repetitively recruited in clinical trials without adequate time off treatment to prevent toxicity and exclude carry-over effects [2].

\section{Sample size}

The sample size is ethically and scientifically important. Too small a sample size may not allow the detection of anything significant and 
may cause type II error (false negative) to occur $[3,11,12]$. On the other hand, too large a sample size raises ethical issues, as subjects are unnecessarily exposed to risk and resources are unnecessarily wasted $[11,12]$.The size of a trial is influenced by the disease to be investigated, the objective of the study, and the study endpoints $[2,3,4]$.

Study endpoints should be chosen to assess drug/vaccine effects that are related to efficacy and safety. Selection of primary endpoint should be based on the primary objective of the trial and should reflect clinically relevant effects. A surrogate endpoint may be used as a primary endpoint when it is likely to predict the clinical outcome. The measurements of the endpoints should be validated prior to use, to ensure the accuracy, precision and reproducibility [2].

In clinical trials involving the comparison of two independent quantitative data sets, the sample size required depends on clinically meaningful difference to be detected, standard deviation of the variable, power, and the nominated significance level. When very small difference in measurement can be detected, it is important to distinguish between statistical significance and clinical significance [11]. In this case, the decision on clinical meaningful difference must be defined.

The efficacy is most convincing when the study can demonstrate the superiority to comparator (placebo or active). However, in some trial, the objective of the trial is to demonstrate that an investigational product is not worse than active comparator, i.e., non-inferiority trial. In other trials, the primary objective may be to show that the responses of different treatments differ by an amount that is clinically unimportant, i.e., equivalence trial [4]. When the objective of the study is to demonstrate non-inferior or equivalence, an equivalence margin should be specified. This margin is the largest difference that can be considered as being clinically acceptable and should be smaller than differences observed in superiority trials of the active comparator [4]. For the equivalence trial (active control), both the upper and the lower equivalence margins are needed; two-sided confidence intervals should be used. Equivalence is concluded when the entire confidence interval falls within the equivalence margins. For the non-inferiority trial, only the lower margin is needed; a one-sided interval should be used. The confidence interval approach has a one-sided hypothesis testing. The null hypothesis is that the treatment difference is equal to the lower equivalence margin versus the alternative that the treatment difference is greater than the lower equivalence margin $[3,4]$.

\section{Ethics Committee Considerations for Ethical Clinical Trial}

Prior ethical review and approval of a clinical trial protocol is a universally required standard $[1,6,7]$. It is therefore important that EC members commit to a timely review, thoroughness, objectivity, competency, and impartiality in review. The members should recognize their conflict of interest and be able to manage it properly. It is vital that EC members maintain confidentiality of the reviewed documents. The dimension of quality review include thoroughness and timely review, safety of the subject, benefit to the subject, appropriateness of study design, ability to identify subject protection measures, equitable, and ability to keeping confidentiality. To demonstrate their competency in ethical review for clinical trial, at a minimum, they should have training in Good Clinical Practice (GCP) and research ethics - human subject protections. When protocols involve children population, at least one of the ethics committee members should be competent in evaluating pediatric ethical, clinical, and psychosocial issues [5].
In reviewing a clinical protocol, EC members should base their decisions on the submitted information, and review the documents with reference to internationally acceptable standards. In deciding if a clinical trial is ethical, the members should consider, but not be limited to, the following important issues:

Scientific merit of the study and the effect of the study on the health of research subjects (i.e., potential harm and benefit)

In ethically acceptable research, risks have been minimized (both by preventing potential harms and minimizing their negative impacts should they occur) and are reasonable in relation to the potential benefits of the study. EC members should recognize that the nature of risks may differ according to the types of clinical trial as well as the locations of the trial to be conducted. For example, risk of pneumonia in Europe is considered lower in comparison to Africa where there is limited health care facility available and accessible, resulting in high mortality. EC members should recognize that risks can occur in different dimensions including physical, social, financial, or psychological. When assessing risks, the probability, duration, and the magnitude of the effect should be taken into account. Furthermore, harm may occur either at an individual level or at the family or population level. Similarly, considerations for benefit should be carried out on different dimensions, taking into account its probability, duration and magnitude. It is important that EC members recognize the limitations of their knowledge and seek external inputs when necessary [9].

\section{Vulnerability of subjects}

EC members must recognize the vulnerability of the subjects in the protocol they review. It is thus important that EC members are aware of the definition of vulnerability. Vulnerable subjects can be describes as individuals whose willingness to volunteer in a clinical trial may be unduly influenced by the expectation of benefits associated with participation, or the fear of a retaliatory response from senior members of a hierarchy in the case of refusal to participate $[1,7]$. Some examples of vulnerable subjects who are members of a group with a hierarchical structure include medical, pharmacy, dentistry, and nursing students, subordinate hospital and laboratory personnel, employees of the pharmaceutical industry, members of the armed forces, and persons kept in detention. Other vulnerable subjects include patients with incurable diseases, persons in nursing homes, unemployed or impoverished persons, patients in emergency situations, ethnic minority groups, homeless persons, migrants, refugees, minors, individuals who are politically powerless, members of communities unfamiliar with modern medical concepts, and those incapable of giving consent. Subjects who have serious, potentially disabling or lifethreatening diseases should be considered as highly vulnerable.

EC members need to assess whether the subjects in the protocol under review is vulnerable and evaluate if the research could be carried out equally well with less vulnerable subjects [7]. EC members need to evaluate if the knowledge gained from research will lead to improved health problems that are characteristics of the vulnerable subjects. EC members must pay attention to the appropriateness of protection that is being proposed by the investigator. For example, the provision of counseling, in the case of HIV study, or the provision of a lawyer, in the case of drug addict subjects, must be considered. The data from the surveys of ECs by Forum for Ethical Review in Asia and Western Pacific (FERCAP: www.fercap-sidcer.org) in the past few years showed that the most common finding in protocol review is the deficiency in recognizing vulnerability of the subjects in research, specifically chronic diseases such as chronic obstructive pulmonary disease, chronic kidney or liver failure, and incurable diseases such as cancer, HIV patients, etc. 


\section{Study design}

EC members should evaluate if the design of the study is appropriate and can yield the desired information. EC members must assess carefully whether the chosen study design is free of possible bias. When there is a control group, randomization and blinding should be used; if not, justification should be provided by the investigator and evaluated by EC members. In some cases where blinding is not possible, EC members should assess its justification.

In the case of placebo control trial as well as fixed dose-dose response design, the design is almost always double blind randomized $[4,12]$. The purpose is to control the placebo effect as well as to control all potential influences on the actual course of the disease. Blinding is intended to minimize the potential biases resulting from differences in management, assessment and interpretation of study results.

\section{Selection of subjects (inclusion/exclusion/withdrawal criteria)}

EC members should assess the appropriateness of subject selection. EC members should assess the inclusion criteria if the chosen population is likely to yield the sought answers. No subject with undue risk or vulnerability should be included in clinical trial unless reasonably justifiable and the risks involved can be satisfactorily managed. EC members should also examine the withdrawal criteria if the criteria have provided sufficient protection for those who may experience unexpectedly high risk as a result of errors in initial evaluation on their risks or adverse effects from the intervention. The criteria should assure EC members that all conditions have been covered and that the subjects will be withdrawn from intervention at an appropriate time to prevent undue risk, particularly in the case of placebo control study [14]

\section{Selection of control group}

EC members should pay particular attention when the investigator proposes the use of placebo or no-treatment as a control group. When evaluating the efficacy of a new drug, the Declaration of Helsinki states that it should be compared with the best current proven intervention. It also states clearly that a placebo may be used only when no current proven intervention exists or when there are compelling and scientifically sound methodological reasons to do so, provided that the patients who receive placebo will not be subject to any serious risk or irreversible harm. From the scientific methodological perspective, some conditions may require the use of placebo comparator to prove absolute efficacy of a new therapy $[4,13]$ (e.g., new drugs for some chronic conditions with waxing and waning symptoms or with high rate of placebo response). The fundamental ethical principle underlying the application of this standard is the avoidance of exploitation, particularly for individuals or communities who may be vulnerable because of their socio-economic status. Extreme care must be taken to avoid abuse of using placebo option [6]. When the trial objective is to measure absolute effect size, placebo control is likely to play a significant role, either alone or in combination with other concurrent control such as active control and/ or dose response [4]. However, when there is effective therapy that is known to prevent death or irreversible morbidity, the use of placebo control is not ethically acceptable $[4,6,7,13,14,16]$.

\section{Sample size}

Clinical trials often involve the comparison of new intervention with the best available treatment or placebo in a sample of subjects, and the difference between the two treatment groups is analyzed using a hypothesis testing. When reviewing sample size, EC members need to assess whether the sample size is large enough to detect a treatment effect (if any) at a given significance level. The common mistake in sample size calculation is that the investigator fails to distinguish between statistical significance and clinical relevance. Another common mistake is the use of one-sided or two-sided testing which can result in different sample size. If the direction of hypothesis testing is known (e.g., non-inferior trial), one-sided testing should be used.

\section{Investigator competence}

The competency of the investigator is vital for ethical research [1] This can be assessed on two dimensions: technical and ethical aspects. The technical competency can be evaluated by education, certification, and experience. In addition to his or hers professional competence, the investigator must have clinical trial competence. This can be assessed from the information presented in the protocol whether the investigator has performed a competent systematic review of current knowledge and previous trials, to be certain that the rational of planned study is justified. For clinical trials, training in GCP and ethics in research is required.

Regarding ethical dimension that relates to appreciation and responsiveness in research, the assessment is limited to the evidence of ethics training, which may not be sufficient to support the competency of investigator in this aspect. However, the history of violating GCP in the past and current trials may guide EC members on the extent of oversights needed.

\section{References}

1. International Conference on Harmonization of Technical Requirements for Registration of harmaceuticals for Human Use: Good Clinical Practice (E6) 10 June 1996

2. International Conference on Harmonization of Technical Requirements for Registration of Pharmaceuticals for Human Use: General Considerations for Clinical Trials (E8), 17 July 1997.

3. International Conference on Harmonization of Technical Requirements for Registration of Pharmaceuticals for Human Use: Statistical Principles for Clinical Trials (E9), 5 February 1998.

4. International Conference on Harmonization of Technical Requirements for Registration of Pharmaceuticals for Human Use: Choice of Control Group and Related Issues in Clinical Trials (E10), 20 July 2000.

5. International Conference on Harmonization of Technical Requirements for Registration of Pharmaceuticals for Human Use: Clinical Investigation of Medicinal Products in Pediatric Population (E11), 20 July 2000.

6. World Medical Association Declaration of Helsinki, Ethical Principles for Medical Research Involving Human Subjects 2008.

7. CIOMS, International Ethical Guidelines for Biomedical Research Involving Human Subjects, 2002.

8. European Textbook on Ethics in Research. Luxembourg: Publications Office of the European Union, 2010

9. WHO Operation guidelines for Ethics Committee in Biomedical Research Geneva: WHO, 2000.

10. Emanuel EJ, Wendler D, Grady C (2000) What makes clinical research ethical? JAMA 283: 2701-2711.

11. Smith FG and Smith JE. Key Topics in Clinical Research: A User Guide to Researching, Analyzing, and Publishing Clinical Data. Oxford: Bios Scientific. Publishers Limited.

12. Piantadosi S (1997) Clinical Trials: A Methodologic Perspective. New York John Wiley \& Sons, Inc.

13. Ellenberg SS, Temple R (2000) Placebo-controlled trials and active-contro trials in the evaluation of new treatment. II. Practical issues and specific cases. Ann Intern Med 133: 464-70 
Citation: Karbwang J, Na-Bangchang K (2013) Practical Application of International Standards and Guidelines to Address Ethical Challenges in Clinical Trials. J Clinic Res Bioeth 4: 144. doi:10.4172/2155-9627.1000144

Page 5 of 5

14. Emanuel EJ, Miller FG (2001) The ethics of placebo-controlled trials--a middle ground N Engl J Med 345: 915-919.

15. Karbwang $\mathrm{J}$ and Torres $\mathrm{C}$. Ethical issues related to clinical trials outside the International Conference on Harmonization regions. Future Med. Chem 3: $1457-1460$
16. Temple R, Ellenberg SS (2000) Placebo-controlled trials and active-contro trials in the evaluation of new treatments. I. Ethical and scientific issues. Ann Intern Med 133: 455-463.

17. Rothman KJ, Michels KB (1994) The continuing unethical use of placebo controls. N Engl J Med 331: 394-398. 